Europhysics Letters

PREPRINT

\title{
Cooperative motion and growing length scales in super- cooled confined liquids
}

\author{
P. SCheidleR ${ }^{1}$, W. KoB ${ }^{2}$ and K. Binder ${ }^{1}$ \\ 1 Institut für Physik, Johannes Gutenberg-Universität, 55099 Mainz, Germany \\ 2 Laboratoire des Verres, Université Montpellier II, 34000 Montpellier, France
}

PACS. 61.20.Lc - Time-dependent properties; relaxation.

PACS. 61.20. Ja - Computer simulation of liquid structure.

PACS. 64.70.Pf - Glass transitions.

\begin{abstract}
Using molecular dynamics simulations we investigate the relaxation dynamics of a supercooled liquid close to a rough as well as close to a smooth wall. For the former situation the relaxation times increase strongly with decreasing distance from the wall whereas in the second case they strongly decrease. We use this dependence to extract various dynamical length scales and show that they grow with decreasing temperature. By calculating the frequency dependent average susceptibility of such confined systems we show that the experimental interpretation of such data is very difficult.
\end{abstract}

Motivation. - The details of the mechanism giving rise to the dramatic slowing down of the dynamics of glass-forming liquids upon supercooling are still unknown (see, e.g. 11). Although the mode-coupling theory of the glass transition allows to rationalize many features of the relaxation dynamics of these systems [2], the answers to certain important questions (e.g. the relaxation dynamics at low temperatures) are still unknown. A further popular approach is the phenomenological concept of "cooperativity", introduced by Kauzmann [3], and Adam and Gibbs [4]. A typical example of this cooperativity is the so-called "cage-effect", i.e. the fact that in a dense liquid each particle is surrounded by neighboring particles which form a temporary cage. In order to allow the particle to change its position the cage has to open up. However, each of the particles of the cage is itself also caged and hence can move only if other particles make room. Therefore one can conclude that the particle motion is collective and there exist "cooperatively rearranging regions" (CRR's) within the liquid. The typical size of a CRR is postulated to grow with decreasing temperature, hence "rationalizing" the slowing down of the dynamics 4,5 . 5 .

Experimentally it is difficult to test the concept of the CRR's since usually one does not have direct access to the dynamics of single particles. Therefore many studies have focused on investigating systems in spatial confinement. If CRR's do exist and grow with decreasing temperature the dynamics should differ from the bulk behavior as soon as the size of the CRR's at a given temperature becomes comparable to the system size. Indeed, almost all experiments on glass formers confined to porous host material [6 12] and supported (or even free standing) films [13 117] do indeed show a relaxation dynamics that differs from the one in the bulk. However, so far it has not been possible to give a conclusive interpretation of

(c) EDP Sciences 
experimental results, since, e.g., one sometimes finds that the dynamics in confined systems is faster than the one of the bulk, whereas in other systems it is slower (see, e.g., [8, 10]). The main reason for the diversity in experimental findings (see, e.g., the review article [18]) is the influence of secondary effects beyond the picture of CRR's: On the one hand density effects can be expected to play a crucial role. Since in experiments only the average density is accessible, one can imagine a situation where the confined liquid shows strong local density variations, e.g. density oscillations due to layering effects, which will strongly influence the dynamics as well. Also the interaction between the surface and the liquid is most likely very important [14, 15]. If the liquid particles are sticking to the wall, their mobility is strongly suppressed and because of cooperativity also the dynamics of particles a certain distance away from the wall will be slowed down, which in turn will influence the dynamic properties of the whole sample. The opposite should hold in a situation where the liquid particles can slip along the boundary.

The goal of the present paper is to use molecular dynamics computer simulations to investigate how these boundary effects influence the relaxation dynamics of confined glass-forming liquids and to what extend growing length scales can be extracted in such systems.

Simulation. - The model liquid under investigation is a binary mixture of particles interacting via a Lennard-Jones (LJ) potential $V_{\alpha \beta}(r)=4 \epsilon_{\alpha \beta}\left[\left(\sigma_{\alpha \beta} / r\right)^{12}-\left(\sigma_{\alpha \beta} / r\right)^{6}\right]$ with $\alpha, \beta \in\{\mathrm{A}, \mathrm{B}\}$, cut-off radii $r_{\alpha, \beta}^{c}=2.5 \cdot \sigma_{\alpha \beta}$ and interaction parameter $\epsilon_{\mathrm{AA}}=1.0, \sigma_{\mathrm{AA}}=1.0$, $\epsilon_{\mathrm{AB}}=1.5, \sigma_{\mathrm{AB}}=0.8, \epsilon_{\mathrm{BB}}=0.5$, and $\sigma_{\mathrm{BB}}=0.88$. In the following we will use $\sigma_{\mathrm{AA}}$ and $\epsilon_{\mathrm{AA}}$ as units of length and energy, respectively, setting Boltzmann's constant $k_{B}=1$, and measure time in units of $\sqrt{m \sigma_{\mathrm{AA}}^{2} / 48 \epsilon_{\mathrm{AA}}}$, where $m$ is the mass of the particles. Previous simulation in the bulk have shown that at low $T$ the dynamics of this mixture quickly slows down and that its mode-coupling temperature $T_{c}$ is around 0.435 19.

We considered two types of walls: a rough and a smooth one. The rough wall was realized by freezing a slice of thickness $2.5 \sigma_{\mathrm{AA}}$ of the LJ liquid and applying the same LJ interaction plus an additional hard core potential to prevent the liquid particles from penetrating into the wall. More details on this type of wall can be found in [21, 22

To mimic a smooth wall at a location $z_{\mathrm{W}}$ we applied an external potential of the form $V_{\alpha \mathrm{W}}(z)=(4 / 45) \pi \rho_{\mathrm{W}} \sigma_{\mathrm{AB}}^{3} \varepsilon_{\alpha \mathrm{W}}\left(\sigma_{\mathrm{AB}} /\left(z-z_{\mathrm{W}}\right)\right)^{9}$ (with $\left.\varepsilon_{\mathrm{AW}}=1.0, \varepsilon_{\mathrm{BW}}=3.0\right)$. By choosing walls at $z_{\mathrm{W}}=-0.65$ and $z_{\mathrm{W}}=15.65$ we make sure that a film width of $D=15.0$ is realized.

The data for the rough wall presented here comes from a simulation of a film with area $L x L$ and thickness $D(L=12.88, D=15.0)$, where periodic boundary conditions are applied in the film plane. It contains $2400 \mathrm{~A}$ and $600 \mathrm{~B}$ particles giving an average density of 1.2 as in the bulk simulations in Ref. [19]. For the smooth wall the area of the film was four times larger. In order to improve the statistics we averaged the results over 16 independent samples. The equations of motion were integrated with the velocity form of the Verlet algorithm, using at low $T$ a time step of 0.02 , and the starting configurations for the microcanonical production runs were carefully equilibrated.

As already mentioned in the previous section it is most important to avoid that the confining walls change the structural properties of the enclosed fluid. This request is by construction fulfilled trivially in the case of the rough wall. For the smooth wall, however, we found that close to the wall strong layering effects occur [22]. To avoid this problem we modified the potential energy of the system by adding a term that coupled directly to the deviation from a constant density profile, i.e. configurations whose profile is not constant (within a certain fluctuation) are energetically disfavored. This modification does indeed allow to obtain a system with an essentially constant density profile and we have checked that its structure is indeed the same as the bulk one [22]). 


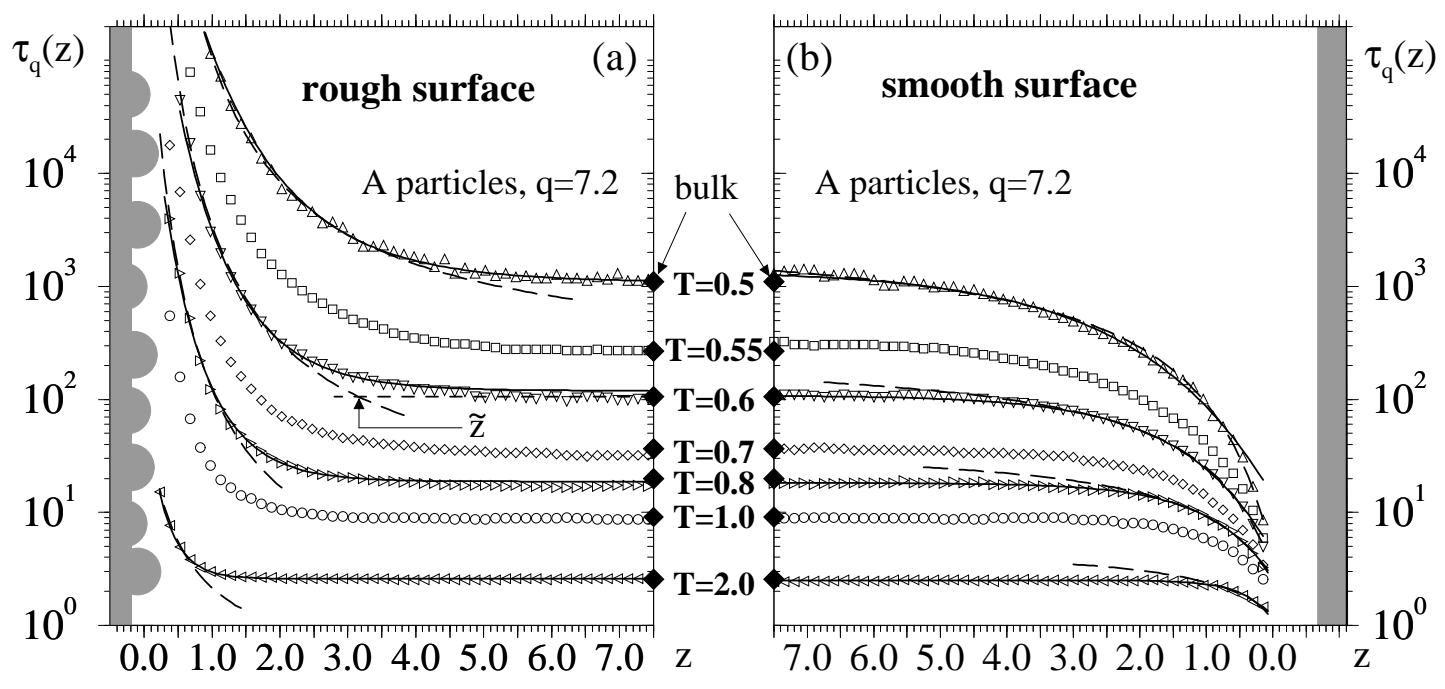

Fig. 1 - Structural relaxation time $\tau_{q}(z)$ at $q=7.2$ as a function of particle distance $z$ from the wall for (a) rough and (b) smooth surface at different temperatures. The large diamonds are the bulk values and the long dashed lines and the solid ones are fits according to Eqs.(伍) and (2), respectively.

Results. - We now discuss the relaxation dynamics of the system as a function of temperature and distance from the wall. Although in the following we focus on the self part of the intermediate scattering function, all the conclusions are also valid for other observables, such as mean squared displacements or the van Hove correlation functions 22 .

From earlier investigations of systems confined between rough surfaces 20.21] we know that the local particle dynamics depends on the distance $z$ from the wall. Therefore we introduce a generalization of the incoherent intermediate scattering function by $F_{\mathrm{s}}(\mathbf{q}, z, t)=N_{\alpha}^{-1} \sum_{j=1}^{N_{\alpha}}$ $\left\langle\exp \left[i \mathbf{q} \cdot\left(\mathbf{r}_{j}(t)-\mathbf{r}_{j}(0)\right)\right] \delta\left(z_{j}(0)-z\right)\right\rangle$, i.e. $F_{\mathrm{s}}(\mathbf{q}, z, t)$ considers only particles that at $t=0$ had a distance $z$ from the wall [21]. We will only present data for A particles and wave vectors q parallel to the wall with $|\mathbf{q}|=7.2$, the location of the maximum in the structure factor. Data for other values of $|\mathbf{q}|$ or the B particles looks qualitatively similar. At low temperatures $F_{\mathrm{s}}(\mathbf{q}, z, t)$ shows a two step relaxation. Hence we can characterize the $\alpha$-relaxation time $\tau_{q}(z)$ by $F_{\mathrm{s}}\left(\mathbf{q}, z, \tau_{q}\right)=e^{-1}$ [20, 21].

In Fig. 1 we show the relaxation times $\tau_{q}(z)$ as a function of $z$ at different temperatures. For particles far away from the wall $F_{\mathrm{s}}(\mathbf{q}, z, t)$ shows bulk behavior and therefore the characteristic relaxation times coincide with the bulk values (filled diamonds). Approaching the rough surface the dynamics is slowed down dramatically, and $\tau_{q}(z)$ grows continuously over several decades in time. In contrast to this the dynamics of the system with smooth surfaces accelerates with decreasing $z$. Furthermore one can see that, in both situations, the region affected by the wall expands with decreasing temperature in that for high $T$ the bulk behavior is realized already at $z \geq 2.0$, while at the lowest $T$ the influence of the wall extends almost to the center of the film. In the following we will use this increase to define a length scale.

In Ref. [20] it was shown that close to the surface the $z$ dependence of $\tau_{q}(z)$ can be described well by the empirical Ansatz

$$
\tau_{q}(z)=f_{q}(T) \exp \left[ \pm \Delta_{q}(T) /\left(z-z_{\mathrm{p}}\right)\right]
$$

with three free parameters $f_{q}(T), z_{\mathrm{p}}=-0.5 \pm 0.15$ and $\Delta_{q}(T)$, the latter weakly temperature 


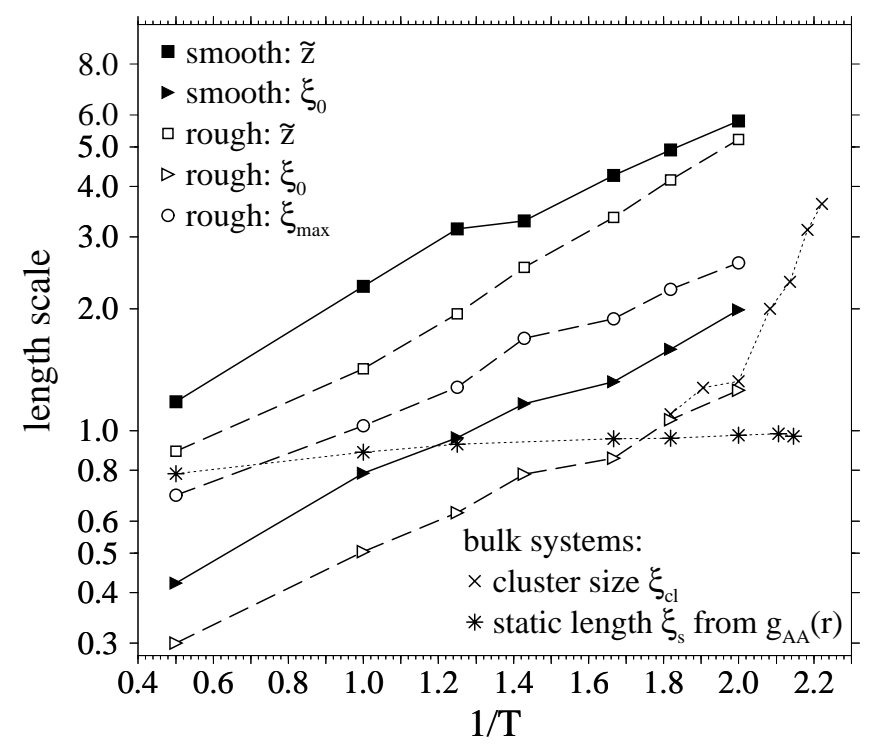

Fig. 2 - Different dynamic length scales (see text for definition) in confined systems as function of inverse temperature in a logarithmic plot. Comparison with static and dynamic length scales in bulk systems. Data for $\xi_{\mathrm{cl}}$ is taken from Ref. 23.

dependent. (Here and in the following the positive (negative) sign corresponds to systems with rough (smooth) surfaces.) The $z$-range for which this fit works well increases with decreasing $T$ (dashed curves in Fig. 11). The location of the crossover to bulk behavior in the center (see data and fits for $T=0.6$ in Fig. 1 1.a) can be used to define a characteristic length scale $\tilde{z}$ whose $T$-dependence will be discussed below.

An alternative Ansatz for the $z$-dependence of $\tau_{q}(z)$ is a function depending on $\exp \left(-z / \xi_{0}\right)$, with a characteristic length scale $\xi_{0}(T)$. We find that the functional form

$$
\ln \left[\left(\tau_{q}(z) / \tau_{q, \infty}\right)^{ \pm 1}\right]=A(T) \cdot \exp \left[-z / \xi_{0}(T)\right]
$$

does indeed describe the $z$-dependence for all values of $z$ (solid lines in Fig. 11). Here $\tau_{q, \infty}$ is the relaxation time of the system in the bulk.

The length scales $\tilde{z}(T)$ and $\xi_{0}(T)$ are obtained directly from $\tau_{q}(z)$. In Ref. [21] we have shown that for the case of the rough surface the whole time and $z$-dependence of $F_{\mathrm{s}}(\mathbf{q}, z, t)$ can be described very well by the Ansatz

$$
F_{\mathrm{S}}(\mathbf{q}, z, t)=F_{\mathrm{s}}^{\mathrm{bulk}}(\mathbf{q}, t) \pm a(t) \exp \left[-(z / \xi(t))^{\beta(t)}\right] .
$$

The time dependence of the length $\xi(t)$ is smooth and shows a maximum, thus showing that the influence of the wall on the dynamics is maximal on the time scale where this maximum occurs (which is on the order of the $\alpha$-relaxation time of the bulk). The value of the maximum in $\xi(t)$ thus allows to define a dynamical length scale $\xi_{\max }$.

In the case of smooth surfaces the Ansatz (3) is not very useful to define a length scale. Although the data is still described reasonably well by Eq. (3), $\xi(t)$ is growing monotonically and therefore it is not possible to read off a $\xi_{\max }$ [22]. 
Fig. 2 is an Arrhenius plot of the $T$-dependence of $\tilde{z}, \xi_{0}$ and $\xi_{\max }$ and from this graph we see that these length scales grow like $\propto \exp [E / T]$. Note that the activation energy $E \approx 1.1$ depends neither on the definition of the length scale nor the type of wall, which gives evidence that the length scales do indeed characterize a length scale intrinsic to the system. Within the $T$-range investigated the growth of this scale is rather small, e.g. $\xi_{0}$ grows only by a factor of 3.5 between $T=2.0$ and $T=0.5$ while bulk relaxation times increase by several orders of magnitude. Furthermore we find no evidence for a divergence at a finite temperature close to the investigated temperature region, in agreement with Ref. [21].

Finally we compare these length scales with the ones that can be identified already in the bulk. Using the van Hove autocorrelation function one can identify the most mobile particles in the system 23. It turns out that these particles are not distributed randomly but instead form temporary clusters (dynamical heterogenieties), the size of which grows with decreasing temperature [23]. This size is included in Fig 2 as well and we find it to be comparable to the dynamic length scale from the present simulation if $T \geq 0.5$.

As an example for a static length scale (in bulk and film systems) we consider the decay length of $g_{\mathrm{AA}}(r)$, the radial distribution function for AA correlations. The envelope of $g_{\mathrm{AA}}(r)-$ 1 is described well by $\exp \left(-r / \xi_{\mathrm{s}}\right)$, which thus defines a length scale $\xi_{\mathrm{s}}$. This length scale shows only a very weak $T$-dependence (Fig.2), in contrast to the dynamical length scales.

Interpretation and Comparison with Experiment. - Having shown how the nature of the walls influences the relaxation dynamics of the particles of the confined system, we now discuss the signature of the observed slowing down/acceleration of the dynamics in an experiment in which the average dynamics of the particles is measured. This is motivated by the fact that in a real experiment it is usually not possible to determine the relaxation dynamics as a function of the distance from the wall whereas the average dynamics is directly accessible.

Thus to compare our simulations with typical experimental data we have to calculate dynamic properties averaged over the whole system, such as $F_{\mathrm{s}}(\mathbf{q}, t)$, the integral of $F_{\mathrm{s}}(\mathbf{q}, z, t)$ over $z$. Although the single curves for $F_{\mathrm{S}}(\mathbf{q}, z, t)$ at different $z$ look very similar for rough and smooth surfaces 20,22 and the $z$-dependence of the relaxation times $\tau_{q}(z)$ has the same functional form we find a qualitative difference in the averaged curves for $F_{\mathrm{s}}(\mathbf{q}, t)$.

For systems with smooth surfaces the $\alpha$-relaxation is described well by a stretched exponential law with a stretching exponent that is slightly lower than the corresponding bulk value, i.e. due to the superposition of different relaxation processes the curves are more stretched.

In contrast to this, $F_{\mathrm{s}}(\mathbf{q}, t)$ for the rough walls shows a long time tail because of the huge relaxation times for particles at the surface [20]. It is possible to describe the whole $\alpha$-relaxation, i.e. also the mentioned tail, by the sum of two stretched exponentials, where the time scale of the slow "process" is about two orders of magnitude larger than the first "process" and the stretching is much more pronounced [22]. Note however, that this is a purely phenomenological description of the data without any underlying physical motivation.

Typically experiments on confined liquids probe the dynamics of the system by measuring the frequency dependence of various susceptibilities (light-and neutron scattering scattering experiments, dielectric measurements). Hence we have calculated the dynamic susceptibility $\chi_{\mathrm{s}}^{\prime \prime}(\mathbf{q}, \omega)=\omega /\left(2 k_{\mathrm{B}} T\right) S_{\mathrm{s}}(\mathbf{q}, \omega)$, where $S_{\mathrm{s}}(\mathbf{q}, \omega)$ is the time-Fourier transform of $F_{\mathrm{s}}(\mathbf{q}, t)$.

In Fig. 3a/b the frequency dependence of $\chi_{\mathbf{s}}^{\prime \prime}(\mathbf{q}, \omega)$ at a low $T$ is shown for bulk systems as well as for films with smooth and rough surfaces. (Note that we show only the frequency range of the $\alpha$-relaxation. The microscopic peak is around $\omega=1$ ). Fig. 3a shows that a smooth surface has a broader $\alpha$-peak than the bulk, in agreement with the observation that in the time domain the stretching exponent is smaller. Furthermore the position of the peak is shifted to slightly higher frequencies, which can be understood from the fact that close 
EUROPHYSICS LETTERS
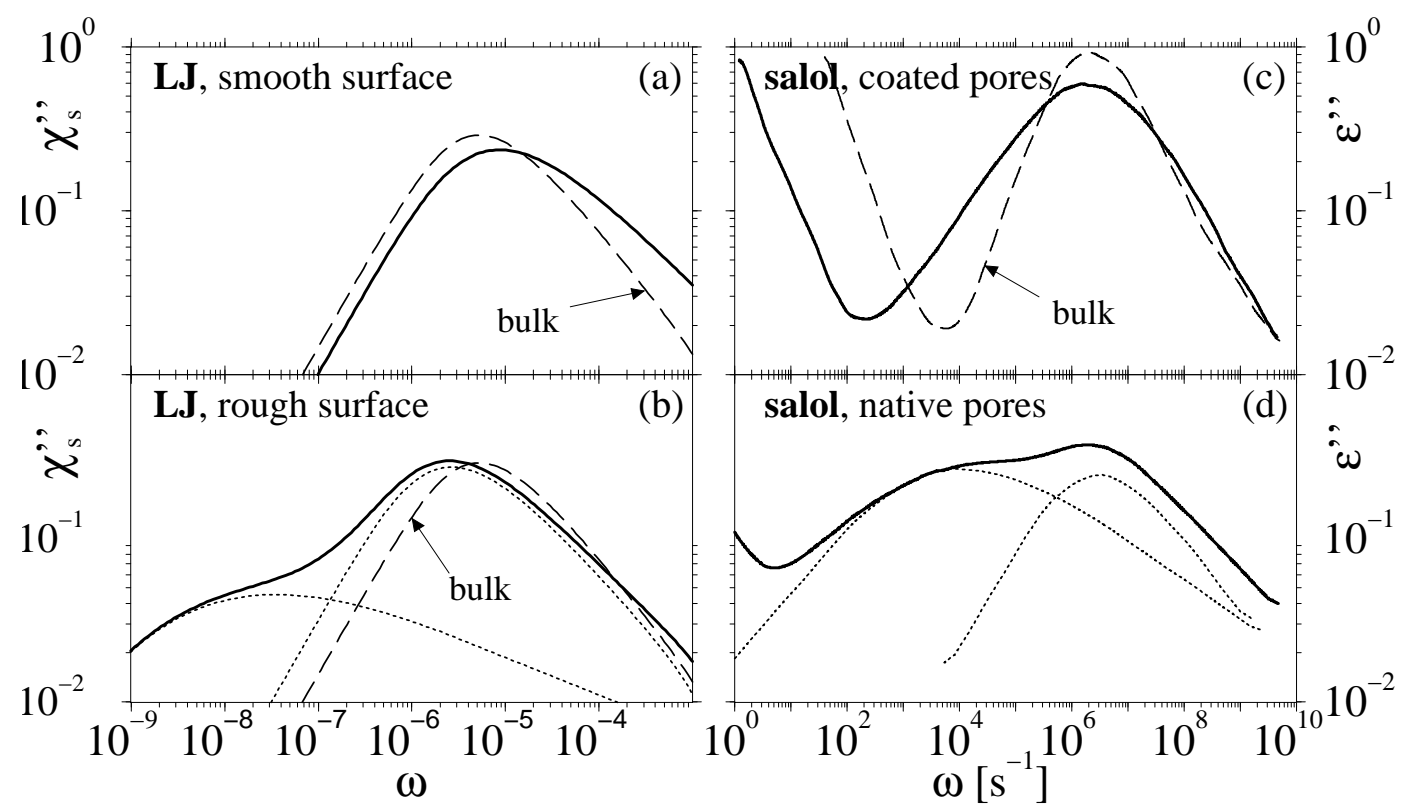

Fig. $3-$ a/b: Dynamic susceptibility $\chi_{\mathrm{s}}^{\prime \prime}(\mathbf{q}, \omega)$ for a LJ liquid in the bulk and confined to a film with smooth and rough surfaces at $q=7.2, T=0.5$. The dotted lines are stretched exponentials. $\mathrm{c} / \mathrm{d}$ : Experimental data for the imaginary part or the dielectric susceptibility of salol in the bulk and confined in pores. The dotted lines are fits with Havriliak-Negami functions. Adapted from Ref. 99.

to the surface the particles move faster than in the bulk. In contrast to these rather small differences between the curve for the bulk and the one for the smooth surface, the spectrum for the system with the rough wall differs strongly from the one of the bulk (Fig. 3b). First of all we note that the location of the peak is shifted to smaller frequencies. More important is, however, that the curve for the rough surface shows a pronounced shoulder left to the $\alpha-$ peak. From Fig. 1 w we know that it would be wrong to ascribe this shoulder to the presence of a second relaxation process, since the relaxation times are a smooth function of $z$. Instead this shoulder is just due to the superposition of a continuum of relaxation processes with very different relaxation times. Also included in the figure are the Fourier-transforms of the two stretched exponentials (dotted lines) which, noted above, describe the whole $\alpha$-relaxation in the time domain.

The same qualitative behavior of relaxation spectra for liquids in confinement is also seen in many experiments. As an example we take dielectric data of the simple glass former salol confined in Vycor glass from Ref. [9]. In this "quasi"-van der Waals liquid H-bonds are mainly of intramolecular nature and therefore the interaction is to a first approximation comparable to the van der Waals system studied here. Samples with uncoated pores, where H-bonds between the molecules and the wall can form, correspond to the situation of a rough surface. If the pore surface is coated, i.e. the formation of the mentioned H-bonds is prevented, the interaction between the wall and the liquid becomes weak, i.e. a smooth surface is realized. In Fig. 3c/d we show the data from Ref. [9] for the bulk, as well as porous systems with a native surface and a treated one. We see that from a qualitative point of view these three spectra are very similar to the ones obtained in our simulation for the three different situations (broadening 
of the peak in the confined system, presence of a shoulder at low $\omega$ in the untreated surface, etc.). (Similar experimental results can be found in Refs. [7, 8]). Also note that the increase of the spectra at low $\omega$ is related to the Maxwell-Wagner polarization of the sample [9] which has nothing to do with the structural relaxation of the system.) We emphasize, however, that for our system the presence of the second peak in the susceptibility is not related to the existence of a layer at the surface that relaxes orders of magnitudes slower than the rest of the liquid, a popular interpretation of such a feature [7 99, 16, 17, 24]. Instead our analysis of the local dynamics has shown that this secondary peak is just the result of averaging over particles that have a continuous and monotonous distribution of relaxation times.

\section{$* * *$}

This work was supported by SFB 262/D1 and BI 314/18 of the DFG. We also thank the HLRZ Jülich for a generous grant of computer time on the T3E.

\section{REFERENCES}

[1] Proceedings of Fourth International Discussion Meeting on Relaxation in Complex Systems, J. Non-Cryst. Solids, to be published (2002).

[2] Götze W., J. Phys.: Condens. Matter, 11 (1999) A1.

[3] Kauzmann W., Chem. Revs., 9 (1948) 219.

[4] Adams G. and Gibbs J.-H., J. Chem. Phys., 43 (1965) 139.

[5] Huth H., Beiner M. And Donth E., Phys. Rev. B, 61 (2000) 15092.

[6] Pissis P., Daoukaki-Diamanti D., Apekis L. and Christodoulides C., J. Phys.: Condens. Matter, 6 (1994) L325.

[7] Barut G., Pissis P., Pelster R. and Nimtz G., Phys. Rev. Lett., 80 (1998) 3543.

[8] Schüller J., Mel'nichenko Yu. B., Richert R. and Fischer E. W., Phys. Rev. Lett., 73 (1994) 2224.

[9] Arndt M., Stannarius R., Groothues H., Hempel H. and Kremer F., Phys. Rev. Lett., 79 (1997) 2077.

[10] Jackson C. L. and McKenna G. B., Chem. Matter, 8 (1996) 2128.

[11] Richert R., Phys. Rev. B, 54 (1996) 15762.

[12] Zorn R., Hartmann L., Frick B., Richter D. and Kremer F., to be published in Ref.[1], (2002) .

[13] Keddie J. L., Jones R. A. L. and Cory R.A., Europhys. Lett., 27 (1994) 59.

[14] Wallace W.E. and van Zanten J. H., Phys. Rev. E, 52 (1995) R3329.

[15] Forrest J. A., Dalnoki-Veress K. and Dutcher J. R., Phys. Rev. E, 56 (1997) 5705.

[16] Forrest J. A. and Mattsson J., Phys. Rev. E, 61 (2000) R53.

[17] Fukao K. and Miyamoto Y., Phys. Rev. E, 61 (2000) 1743.

[18] McKenna G. B., J. Phys. France IV, 10, Pr7 (2000) 53.

[19] Kob W. and Andersen H.-C., Phys. Rev. E, 51 (1995) 4626, Phys. Rev. E, 52 (1995) 4134

[20] Scheidler P., Kob W. and Binder K., Europhys. Lett., 52 (2000) 277.

[21] Scheidler P., Kob W., Binder K. and Parisi G., Phil. Mag. B, 82 (2002) 283.

[22] Scheidler P., Kob W. and Binder K., to be published, (2002).

[23] Donati C., Douglas J. F., Kob W., Plimpton S. J., Poole P. H. and Glotzer S. C., Phys. Rev. Lett., 80 (1998) 2338.

[24] Gallo P., Rovere M. and Spohr E., Phys. Rev. Lett., 85 (2000) 4317. 